\title{
Insegurança alimentar e nutricional de famílias usuárias da Estratégia Saúde da Família em dois municípios paraibanos, Brasil
}

\author{
Food and nutritional insecurity of families using the Family Health \\ Strategy in two municipalities in Paraíba, Brazil
}

Eduarda Emanuela Silva dos Santos (http://orcid.org/0000-0002-3369-8930) ${ }^{1}$

Maria Mônica de Oliveira (http://orcid.org/0000-0002-2775-4318) ${ }^{1}$

Ítalo de Macedo Bernardino (http://orcid.org/0000-0003-4750-5666) ${ }^{1}$

Dixis Figueroa Pedraza (http://orcid.org/0000-0002-5394-828X) ${ }^{1}$
${ }^{1}$ Universidade Estadual da Paraíba. R. Baraúnas 351, Bodocongó. 58109-753, Campina Grande PB Brasil. dudanutri@hotmail.com

\begin{abstract}
The objective of this article is to evaluate factors associated with food and nutritional insecurity in families with children under 5 years of age living in areas covered by the Family Health Strategy. Cross-sectional study involving 406 families from two municipalities in the Metropolitan Region of João Pessoa, Paraíba. The Brazilian Food Insecurity Scale was used to assess the families' food and nutritional security. The determinants of moderate/severe food insecurity were analyzed using the Decision Tree. Food and nutritional insecurity reached $71.4 \%$ of families. Moderate/severe food insecurity (32\%) was primarily associated with the benefit of the Family Allowance (Bolsa Familia) Program, and also with family composition consisting of children under 2 years of age, lower socioeconomic status, and family dysfunction. The results showed high prevalence of food and nutritional insecurity whose more serious levels suggest the importance of interventions aimed at improving the Family Allowance Program for the conditions of households with children under 2 years of age, socioeconomic situation of families, and functionality of families.
\end{abstract}

Key words Primary Health Care, Child, Food and nutrition security, Socioeconomic factors, Government programs
Resumo O objetivo deste artigo é avaliar fatores associados à insegurança alimentar e nutricional de famílias com crianças menores de 5 anos de idade residentes em áreas de abrangência da Estratégia Saúde da Família. Estudo transversal que envolveu 406 famílias de dois municípios da Região Metropolitana de João Pessoa, Paraíba. Para a avaliação da segurança alimentar e nutricional das famílias foi utilizada a Escala Brasileira de Insegurança Alimentar. Os determinantes da insegurança alimentar moderada/grave foram analisados por meio de Árvore de Decisão. A insegurança alimentar e nutricional atingiu $71,4 \%$ das famílias. A insegurança alimentar moderada/ grave (32\%) associou-se, primeiramente, ao benefício do Programa Bolsa Família, além de à família constituída por crianças menores de 2 anos, ao menor nível socioeconômico e à disfunção familiar. Os resultados mostram prevalências elevadas de insegurança alimentar e nutricional, cujos graus mais graves sugerem a importância de intervenções direcionadas a melhorias do Programa Bolsa Família, nas condições dos domicílios com crianças menores de 2 anos, na situação socioeconômica das famílias e na funcionalidade familiar. Palavras-chave Atenção Primária à Saúde, Criança, Segurança alimentar e nutricional, Fatores socioeconômicos, Programas governamentais 


\section{Introdução}

O acesso à alimentação deve ser considerado um direito humano a ser promovido por meio de políticas públicas que prestem atenção à Segurança Alimentar e Nutricional (SAN) com prioridade nas pessoas mais vulneráveis como as crianças menores de 5 anos $^{1}$. A violação desse direito, como importante problema estrutural da sociedade, ameaça as condições de saúde e nutrição da população ${ }^{2}$. Diante de sua importância, a promoção da SAN foi colocada como um dos objetivos globais do desenvolvimento sustentável, do qual o Brasil é signatário ${ }^{3}$.

Dados da Organização das Nações Unidas para a Alimentação e a Agricultura revelaram que em 2017 aproximadamente 821 milhões de pessoas no mundo, cerca de uma em cada nove, foram incapazes de satisfazer suas necessidades básicas de alimentos, representando um aumento de 17 milhões apenas em um ano. Na América Latina e no Caribe, a estimativa foi de 39,3 milhões de pessoas, representando um aumento de 400 mil pessoas desde $2016^{1}$. No Brasil, a Pesquisa Nacional por Amostra de Domicílios (PNAD), utilizando a Escala Brasileira de Insegurança Alimentar (EBIA), identificou 22,6\% dos domicílios com algum grau de Insegurança Alimentar e Nutricional (IAN), destacando-se o Norte $(36,1 \%)$ e o Nordeste $(38,1 \%)$ do país com as piores condições. No Estado da Paraíba, segundo a mesma pesquisa, $41 \%$ dos domicílios estavam em IAN, sendo $17,5 \%$ moderada/grave 4 .

A IAN é reconhecida como um fenômeno complexo e multidimensional determinado, principalmente, pela pobreza e pelas desigualdades sociais, trazendo repercussões negativas nos grupos mais vulneráveis ${ }^{5,6}$. Nesse contexto, os Programas de Transferência Condicionada de Renda (PTCR) são considerados estratégias de proteção social de combate à pobreza e à fome, que incluem condicionalidades de participação associadas a benefícios na nutrição, saúde e educação ${ }^{7}$. Os efeitos positivos desses programas na SAN e no acesso à saúde são sugeridos na literatura. Entretanto, há também controvérsias em relação aos impactos na situação vacinal, na diversidade alimentar, no estado de saúde e no crescimento da criança. Revela-se, assim, que as vias de impacto dos PTCR ainda não estão bem definidas ${ }^{8}$.

No Brasil, o Programa Bolsa Família (PBF) tem mostrado benefícios na SAN, no entanto sem repercutir necessariamente na qualidade nutricional ${ }^{9}$. Ainda, os resultados de estudos que comparam famílias beneficiárias e sem o benfício do Programa não são consistentes. Mostra-se, por um lado, o adequado direcionamento dos recursos, contudo sem melhorias na SAN. Na outra direção, sugere-se que a correta focalização do PBF impacte positivamente a SAN, na medida em que atinge as famílias de maior vulnerabilidade social ${ }^{6}$. Nesse sentido, uma revisão sistemática da literatura mostrou chances aumentadas de IAN em populações que utilizam os serviços públicos de saúde/beneficiários do PBF. Destacase, ainda, que as pesquisas sobre SAN no Brasil precisam de maior dinamismo e integração entre os focos econômico e social ${ }^{5}$.

Apesar do apoio social ser um dos determinantes da IAN ${ }^{10}$, seu estudo tem sido pouco explorado na literatura científica ${ }^{11}$. Até onde é de conhecimento dos autores, apenas dois artigos foram publicados contemplando os resultados de alguma análise nesse sentido com população brasileira $^{11,12}$.

Identificar as famílias mais vulneráveis à IAN e seus fatores associados é primordial para a avaliação das condições de vida e o planejamento de intervenções de promoção à saúde e combate à fome ${ }^{6}$. Nesse sentido, famílias com crianças menores de 5 anos representam um grupo de marcada suscetibilidade. As crianças podem, ainda, ter sua capacidade produtiva reduzida no futuro como consequência da transmissão intergeracional da desigualdade socioeconômica ${ }^{13}$.

Nesse contexto, o objetivo deste trabalho foi avaliar fatores associados à IAN de famílias com crianças menores de 5 anos de idade residentes em áreas de abrangência da Estratégia Saúde da Família, em municípios priorizados com recursos financeiros para a área de nutrição.

\section{Métodos}

Trata-se de um estudo transversal realizado com famílias residentes em dois municípios do Estado da Paraíba, atendidas na Estratégia Saúde da Família (ESF), com crianças de 0 a 59 meses de idade no núcleo familiar. Esses municípios foram escolhidos por serem prioritários para ações voltadas à prevenção do sobrepeso infantil ${ }^{14}$. Além disso, considerou-se semelhanças entre os mesmos em relação à posição geográfica (localização na região metropolitana da capital do estado da Paraíba, com acesso à rede de serviços nela disponibilizados), indicadores sociais (índice de desenvolvimento humano de nível médio) e cobertura da ESF (100\%). Segundo o porte populacional, 
o município de Bayeux tem população total de 99.716 habitantes e 7.862 crianças menores de 5 anos, das quais 4.514 são beneficiárias do PBF. A população de Cabedelo é composta por 57.994 indivíduos e 4.596 crianças menores de 5 anos, das quais 2.553 têm o benefício do PTCR. Ambos os municípios possuem três Núcleos de Apoio à Saúde da Família (NASF), com 28 e 20 equipes da ESF, respectivamente, com arranjos tanto de equipes convencionais quanto do Programa Mais Médicos (PMM).

Selecionaram para participar da pesquisa 12 equipes de cada município, seis convencionais e a mesma quantidade do PMM. As mesmas foram selecionadas aleatoriamente dentre aquelas vinculadas ao mesmo NASF.

Para o cálculo do tamanho amostral adotouse a prevalência de insegurança alimentar moderada/grave de 31,6\% entre famílias que usam serviços públicos de saúde/beneficiários do $\mathrm{PBF}^{5}$. Considerando população $=12.458$, erro amostral máximo de 5\% sob nível de significância de 95\%, $25 \%$ para compensar eventuais perdas e controle de fatores de confusão, estimou-se a necessidade de estudar 431 famílias (18 famílias/equipe).

As famílias foram selecionadas de forma aleatória por ocasião do atendimento de rotina ou puericultura da criança menor de 5 anos, até completar o número definido para cada equipe. Em caso de equipes em que os atendimentos às crianças eram reduzidos, foi realizada busca ativa nos domicílios, com base nos registros das equipes, sendo as famílias selecionadas de forma aleatória.

A coleta de dados foi realizada nas unidades de saúde, no segundo semestre de 2017, por pesquisadores entrevistadores (profissionais e estudantes da área de saúde) com experiência prévia em trabalho de campo, o qual foi supervisionado por profissional capacitado. O controle de qualidade do estudo incluiu: treinamento e padronização dos entrevistadores, construção de Manual de Instruções e realização de estudo piloto no município de Campina Grande.

$O$ instrumento de coleta de dados incluiu o diagnóstico da SAN familiar (variável dependente) e a aplicação de um questionário estruturado às mães das crianças contendo informações relacionadas a: i. perfil materno-infantil, (sexo e idade da criança, idade gestacional, peso ao nascer, estado nutricional antropométrico da mãe); ii. acesso a serviços de saúde (trimestre de início do pré-natal, número de consultas de pré-natal, tipo de equipe de saúde em relação à atuação de nutricionista) e programas sociais (frequência da criança à creche, benefício do PBF); iii. apoio social (convivência da mãe com companheiro, funcionalidade familiar), e iv. situação socioeconômica familiar (trabalho da mãe fora de casa, classificação socioeconômica). A idade, a idade gestacional e o peso ao nascer da criança foram obtidos por meio da informação materna e/ou consulta à Caderneta de Saúde da Criança. Foram classificadas com prematuridade e/ou baixo peso ao nascer crianças nascidas antes da $37^{\mathrm{a}}$ semana de gestação e/ou com peso ao nascer < $2.500 \mathrm{~g}$. O pré-natal foi definido como inadequado se iniciado após o primeiro trimestre da gravidez e o número de consultas foi inferior a seis ${ }^{15}$.

$\mathrm{Na}$ avaliação do estado nutricional das mães foram utilizados equipamentos e técnicas padronizados ${ }^{16}$. A estatura foi aferida usando estadiômetro (WCS ${ }^{\circledR}$ ) com escala em milímetros ( $\mathrm{mm}$ ). O peso foi aferido utilizando-se balança eletrônica do tipo plataforma com capacidade para $150 \mathrm{~kg}$ e graduação em 100g (Tanita UM-080 ${ }^{\circledR}$ ). Todas as medidas foram realizadas duas vezes e a média foi utilizada para fins de registro. A baixa estatura foi definida pelo ponto de corte 155,0 cm que corresponde ao percentil cinco da relação estatura para idade, considerando a idade de 20 anos ou mais, segundo o National Center for Health Statistic (CDC Growth Charts. http://www. cdc.gov/growthcharts/cdc_charts.htm, acessado em 18/Set/2018). O Índice de Massa Corporal foi calculado pela razão entre o peso $(\mathrm{kg})$ e a estatura (metros) ao quadrado, utilizando-se os seguintes pontos de corte: baixo peso $(<18,5)$, adequado $\left(18,5\right.$ - 24,9) e sobrepeso/obesidade $(\geq 25)^{16}$.

Para avaliar a funcionalidade familiar, utilizou-se o questionário APGAR Familiar ${ }^{17}$. Esse instrumento é composto por cinco questões, uma para cada domínio de avaliação: adaptation (adaptação), o qual compreende os recursos familiares oferecidos quando se faz necessária assistência; partnership (companheirismo), que se refere à reciprocidade nas comunicações familiares e na solução de problemas; growth (desenvolvimento), relativo à disponibilidade da família para mudanças de papéis e desenvolvimento emocional; affection (afetividade), compreendendo a intimidade e as interações emocionais no contexto familiar, e resolve (capacidade resolutiva), que está associada à decisão, determinação ou resolutividade em uma unidade familiar. O questionário apresenta três opções de respostas (sempre, que equivale a dois pontos; algumas vezes, a um ponto, e nunca), com pontuação total que varia de 0 a 10 . As famílias com pontuação de 0 a 3 são classificadas com elevada disfunção 
familiar; de 4 a 6, moderada disfunção familiar, e de 7 a 10, boa funcionalidade familiar ${ }^{17,18}$.

A classificação socioeconômica da família baseou-se nos critérios da Associação Brasileira de Empresas de Pesquisa ${ }^{19}$, que é usado para estimar o poder de compra de famílias brasileiras. Para esta classificação considera-se a existência de vaso sanitário no domicílio, a contratação de empregada doméstica, a posse de bens, o grau de instrução do chefe de família e o acesso a serviços públicos. As famílias foram classificadas nos seguintes classes: alta (pontuação de 29 a 100), intermediária (pontuação de 17 a 28) e baixa (pontuação de 0 a 16). Para fins de análise, as classes alta e intermediária foram consideradas em conjunto.

A situação de SAN das famílias foi avaliada por meio da EBIA com 14 itens ${ }^{20}$. Esta é uma escala validada que possibilita classificar as famílias em diferentes graus de insegurança alimentar: segurança alimentar quando todas as questões são respondidas de forma negativa; insegurança alimentar leve quando tem-se até cinco questões com respostas positivas; insegurança alimentar moderada nos casos de seis a nove respostas positivas e insegurança alimentar grave para 10 a 14 respostas positivas.

O grupo de variáveis independentes foi formado pelas seguintes características: sexo e idade da criança; prematuridade e/ou baixo peso ao nascer; estatura e Índice de Massa Corporal materno; adequação do pré-natal; tipo de equipe de saúde da ESF (com ou sem nutricionista); frequência da criança à creche, benefício do PBF; convivência da mãe com companheiro; funcionalidade familiar; trabalho da mãe fora de casa; classificação socioeconômica da família e município de residência. A SAN foi a variável dependente do estudo, dicotomizada em segurança alimentar/insegurança alimentar leve e insegurança alimentar moderada/grave.

Os dados coletados foram organizados em planilhas eletrônicas e digitados em dupla entrada em um banco de dados customizado com verificações de consistência e restrições de intervalo. Utilizou-se para as análises estatísticas o banco produzido após verificação de análise de consistência dos dados digitados.

Inicialmente, foram calculadas as frequências absolutas e os percentuais de todas as variáveis em estudo. Em seguida, foi realizada a análise bivariada para testar a associação entre o nível de SAN e todas as variáveis independentes utilizando o Teste Qui-quadrado. Para explicar a insegu- rança alimentar moderada/grave, inseriram-se todas as variáveis em um modelo de Árvore de Decisão por meio do algoritmo CHAID.

A Árvore de Decisão consiste em regras de decisão que realizam sucessivas divisões em um conjunto de dados, de modo a torná-lo cada vez mais homogêneo no tocante à variável dependente. A técnica facilita a identificação das variáveis mais relevantes para a descrição de um problema e de relações hierárquicas, o que pode ser utilizado para apoiar decisões relativas à saúde. Para esses fins, descrevem-se de forma gráfica os principais componentes de um problema: o modelo propriamente dito, as probabilidades de ocorrência dos eventos modelados e os valores dos desfechos que existem no final de cada percurso. A análise tem a capacidade de segmentar eficientemente as populações em subconjuntos significativos, permitindo a identificação de segmentos marginalizados, o que pode ser usado para o direcionamento efetivo de recursos e de medidas de intervenção. Dessa forma, possibilita inferências que não são admissíveis baseando-se nos resultados do modelo de regressão em que as decisões são voltadas para o membro médio da população, sem considerar os subgrupos de população como o principal alvo ${ }^{21,22}$.

A Árvore de Decisão foi construida utilizando um gráfico que começa com um nó raiz, em que todas as observações da amostra são apresentadas. Os nós produzidos em sequência representam subdivisões dos dados em grupos cada vez mais homogêneos, sendo denominados de nós-filhos. Quando não há mais possibilidade de divisão, obtem-se os nós-terminais ou folhas ${ }^{23}$. $\mathrm{O}$ modelo foi ajustado mediante sucessivas divisões binárias (nós) nos conjuntos de dados. O critério de parada adotado foi o valor $\mathrm{p}<0,05$ da estatística $\chi^{2}$ usando a correção de Bonferroni. $O$ ajuste do modelo final foi avaliado pela estimativa de risco geral, que compara a diferença entre o valor esperado e o observado pelo modelo, indicando em que medida a árvore corretamente prediz os resultados. Só permaneceram no gráfico final as variáveis que obtiveram valor $\mathrm{p}$ ajustado $<0,05$.

As análises estatísticas foram feitas no programa SPSS (Statistical Package for Social Sciences) versão 20.0 (SPSS Inc., Chicago, Estados Unidos).

O Projeto foi aprovado pelo Comitê de Ética em Pesquisa da Universidade Estadual da Paraíba. Todos os participantes da pesquisa assinaram o Termo de Consentimento Livre e Esclarecido, condição necessária à participação no estudo. 


\section{Resultados}

Participaram do estudo 406 famílias com crianças menores de 5 anos. De acordo com a EBIA, $71,9 \%$ das famílias apresentaram algum grau de IAN, sendo 75,9\% no município de Bayeux e 67,0\% no município de Cabedelo. Em Bayeux, as prevalências de insegurança alimentar leve, moderada e grave foram de $38,8 \%, 20,6 \%$ e $16,5 \%$, respectivamente; enquanto que em Cabedelo foram de $41,2 \%, 13,4 \%$ e $12,4 \%$. Foram diagnosticadas com insegurança alimentar moderada/ grave $32 \%$ das famílias (Figura 1).

Do total de famílias, $247(60,8 \%)$ tinham no seu núcleo menores de 2 anos de idade e 208 $(51,2 \%)$ crianças do sexo masculino. Quanto ao estado nutricional materno, evidenciou-se prevalências expressivas de déficits de estatura $(35,5 \%)$ e de peso $(50,5 \%)$, assim como de sobrepeso/obesidade $(13,6 \%)$. A maioria das famílias foi classificada como beneficiária do PBF $(60,1 \%)$, altamente funcional $(67,0 \%)$ e de classe socioeconômica baixa $(64,0 \%)$ (Tabela 1 ).

Em relação às análises bivariadas (Tabela 1), observou-se que ter nascido prematuro e/ou com baixo peso ao nascer $(p=0,027)$, o pré-natal inadequado $(p=0,018)$ e a ausência de nutricionista nas equipes de saúde $(p=0,016)$ representaram exposições associadas à insegurança alimentar moderada/grave. Em relação aos programas sociais, ao apoio social e à situação socioeconô- mica, prevalências maiores de insegurança alimentar moderada/grave foram encontradas nos seguintes casos: criança que frequenta creche, benefício do PBF, família classificada como de disfunção grave, mãe que não trabalha fora de casa e classe socioeconômica baixa. Adicionalmente, a insegurança alimentar moderada/grave foi mais frequente entre famílias residentes no município de Bayeux.

O modelo final da Árvore de Decisão foi construído por 13 nós, conforme mostrado na Figura 2. Segundo o modelo, a variável que mais interferiu na presença de insegurança alimentar moderada/grave foi o recebimento do $\mathrm{PBF}$ ( $\mathrm{p}<$ 0,001), sendo observada prevalência de 41,8\% do desfecho entre as famílias beneficiárias e de $17,3 \%$ entre as que não têm o benefício. Para as famílias que usufruíam do benefício, a presença de criança menor de 2 anos no núcleo familiar exerceu importante influência na prevalência de insegurança alimentar moderada/grave $(\mathrm{p}=$ $0,044)$ e, nestas, a funcionalidade familiar caracterizada como disfunção grave $(\mathrm{p}=0,008)$ também representou um fator relevante. A influência do município de residência restringiuse às famílias com crianças de 2 anos ou mais. Para as famílias não beneficiárias do PBF, as de nível socioeconômico baixo alcançaram maior prevalência de insegurança alimentar moderada/ grave $(p=0,023)$. Além disso, no nó-filho para a melhor condição socioeconômica a funciona-

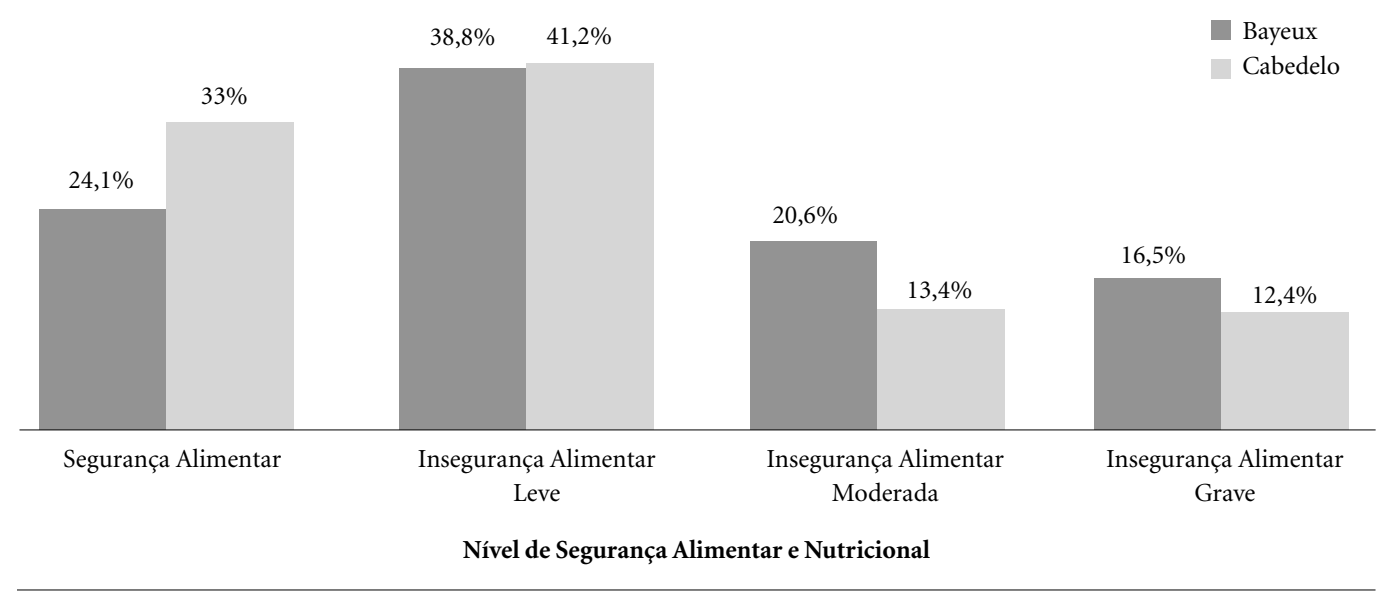

Figura 1. Prevalências de Segurança Alimentar e Nutricional em famílias com crianças menores de cinco anos residentes em dois municípios do estado da Paraíba, 2017. 
Tabela 1. Situação de Segurança Alimentar e Nutricional, de acordo com o perfil materno-infantil, acesso a serviços de saúde e programas sociais, apoio social e situação socioeconômica, em famílias com crianças menores de cinco anos residentes em dois municípios do estado da Paraíba, 2017.

\begin{tabular}{|c|c|c|c|c|}
\hline \multirow{3}{*}{ Variáveis } & \multirow{3}{*}{$\begin{array}{l}\text { Total } \\
\mathbf{n}(\%)\end{array}$} & \multicolumn{2}{|c|}{ Segurança Alimentar e Nutricional } & \multirow{3}{*}{ p-valor } \\
\hline & & Nível A & Nível B & \\
\hline & & n (\%) & n (\%) & \\
\hline \multicolumn{5}{|l|}{ Perfil materno-infantil } \\
\hline Idade da criança (anos) & & & & 0,843 \\
\hline$\geq 2$ & $159(39,2)$ & $109(68,6)$ & $50(31,4)$ & \\
\hline$<2$ & $247(60,8)$ & $167(67,6)$ & $80(32,4)$ & \\
\hline Sexo da criança & & & & 0,349 \\
\hline Masculino & $208(51,2)$ & $137(65,9)$ & $71(34,1)$ & \\
\hline Feminino & $198(48,8)$ & $139(70,2)$ & $59(29,8)$ & \\
\hline Prematuridade e/ou baixo peso ao nascer & & & & 0,027 \\
\hline Não & $329(81,4)$ & $232(70,5)$ & $97(29,5)$ & \\
\hline $\begin{array}{l}\text { Sim (nascimento antes da } 37^{\mathrm{a}} \text { semana de } \\
\text { gestação e/ou com peso }<2.500 \mathrm{~g} \text { ) }\end{array}$ & $75(18,6)$ & $43(57,3)$ & $32(42,7)$ & \\
\hline Estatura da mãe $(\mathrm{cm})$ & & & & 0,190 \\
\hline Adequada & $262(64,5)$ & $184(70,2)$ & $78(29,8)$ & \\
\hline Inadequada $(<155,0)$ & $144(35,5)$ & $92(63,9)$ & $52(36,1)$ & \\
\hline Índice de Massa Corporal da mãe $\left(\mathrm{Kg} / \mathrm{m}^{2}\right)$ & & & & 0,413 \\
\hline Adequado & $146(35,9)$ & $105(71,9)$ & $41(28,1)$ & \\
\hline Déficit de peso $(<18,5)$ & $205(50,5)$ & $136(66,3)$ & $69(33,7)$ & \\
\hline Sobrepeso/obesidade $(\geq 25)$ & $55(13,6)$ & $35(63,6)$ & $20(36,4)$ & \\
\hline \multicolumn{5}{|l|}{ Acesso a serviços de saúde } \\
\hline Adequação do início do pré-natal & & & & 0,018 \\
\hline Adequado & $300(75,2)$ & $214(71,3)$ & $86(28,7)$ & \\
\hline $\begin{array}{l}\text { Inadequado (iniciado após o primeiro trimestre } \\
\text { da gravidez e número de consultas }<6 \text { ) }\end{array}$ & $99(24,8)$ & $58(58,6)$ & $41(41,4)$ & \\
\hline Tipo de equipe de saúde & & & & 0,016 \\
\hline Com nutricionista & $182(44,8)$ & $135(74,2)$ & $47(25,8)$ & \\
\hline Sem nutricionista & $224(55,2)$ & $141(62,9)$ & $83(37,1)$ & \\
\hline \multicolumn{5}{|l|}{ Acesso a programas sociais } \\
\hline Frequência da criança a creche & & & & 0,039 \\
\hline Sim & $131(32,3)$ & $80(61,1)$ & $51(38,9)$ & \\
\hline Não & $275(67,7)$ & $196(71,3)$ & $79(28,7)$ & \\
\hline Benefício do Programa Bolsa Família & & & & $<0,001$ \\
\hline Sim & $244(60,1)$ & $142(58,2)$ & $102(41,8)$ & \\
\hline Não & $162(39,9)$ & $134(82,7)$ & $28(17,3)$ & \\
\hline \multicolumn{5}{|l|}{ Apoio social } \\
\hline Convivência da mãe com companheiro & & & & 0,058 \\
\hline Sim & $308(75,9)$ & $217(70,5)$ & $91(29,5)$ & \\
\hline Não & $98(24,1)$ & $59(60,2)$ & $39(39,8)$ & \\
\hline Funcionalidade familiar & & & & $<0,001$ \\
\hline Altamente funcional & $272(67,0)$ & $202(74,3)$ & $70(25,7)$ & \\
\hline Disfunção moderada & $97(23,9)$ & $56(57,7)$ & $41(42,3)$ & \\
\hline Disfunção grave & $37(9,1)$ & $18(48,6)$ & $19(51,4)$ & \\
\hline \multicolumn{5}{|l|}{ Situação socioeconômica } \\
\hline Trabalho materno fora de casa & & & & 0,021 \\
\hline $\operatorname{Sim}$ & $108(26,6)$ & $83(76,9)$ & $25(23,1)$ & \\
\hline Não & $298(73,4)$ & $193(64,8)$ & $105(35,2)$ & \\
\hline
\end{tabular}


Tabela 1. Situação de Segurança Alimentar e Nutricional, de acordo com o perfil materno-infantil, acesso a serviços de saúde e programas sociais, apoio social e situação socioeconômica, em famílias com crianças menores de cinco anos residentes em dois municípios do estado da Paraíba, 2017.

\begin{tabular}{|c|c|c|c|c|}
\hline \multirow{3}{*}{ Variáveis } & \multirow{3}{*}{$\begin{array}{c}\text { Total } \\
\mathbf{n}(\%) \\
\end{array}$} & \multicolumn{2}{|c|}{ Segurança Alimentar e Nutricional } & \multirow{3}{*}{ p-valor } \\
\hline & & Nível A & Nível B & \\
\hline & & n (\%) & n (\%) & \\
\hline Classificação socioeconômica da família & & & & $<0,001$ \\
\hline Classe intermediária/alta & $146(36,0)$ & $121(82,9)$ & $25(17,1)$ & \\
\hline Classe baixa & $260(64,0)$ & $155(59,6)$ & $105(40,4)$ & \\
\hline Município de residência & & & & 0,016 \\
\hline Bayeux & $224(55,2)$ & $141(62,9)$ & $83(37,1)$ & \\
\hline Cabedelo & $182(44,8)$ & $135(74,2)$ & $47(25,8)$ & \\
\hline
\end{tabular}

Nível A: Segurança alimentar/insegurança alimentar leve, Nível B: Insegurança alimentar moderada/grave, p-valor: valor de significância estatística segundo o Teste Qui-quadrado; valor de $\mathrm{p}<0,05$.

lidade familiar influenciou a prevalência de insegurança alimentar moderada/grave $(\mathrm{p}=0,001)$, com prevalência de $38,5 \%$ entre as famílias com disfunção moderada/grave e apenas $5,3 \%$ entre as altamente funcionais.

\section{Discussão}

Com o objetivo de identificar fatores associados à IAN, este estudo utilizou um modelo multivariado de Árvore de Decisão. Com base no diagrama de árvore, foi possível identificar subgrupos de famílias com perfis específicos que deveriam ser alvo de estratégias de fortalecimento da SAN, destacando-se a importância de reforçar a atenção entre os beneficiários do PBF. Nesse grupo, as famílias com crianças menores de 2 anos, e nestas as caracterizadas por menor suporte social, representaram distinções importantes para as decisões relacionadas aos processos de focalização. Cabe ressaltar a facilidade de identificar as famílias com tais características. Este trabalho abre perspectivas para novos estudos utilizando abordagens preditivas sobre a IAN e seus determinantes sociais, por meio da adoção de técnicas de ciência de dados aplicadas à saúde.

A prevalência de IAN $(71,4 \%)$ na população estudada foi maior do que a reportada nacionalmente na Pesquisa Nacional por Amostra de Domicílios $(22,6 \%)^{4}$. Entretanto, é próxima da reportada em famílias com crianças menores de 5 anos do Estado do Maranhão $(70,4 \%)^{24}$ e do município de Campina Grande-PB $(69,2 \%)^{6}$. Esse parece não ser apenas um problema de saúde pública do Brasil, revelando-se, por exemplo, prevalência similar de IAN no México $(75,7 \%)^{25}$ e no sul da Etiópia $(75,8 \%)^{26}$. Para a insegurança alimentar moderada/grave, a prevalência registrada na atual pesquisa (32\%) é superior à observada nacionalmente $(7,8 \%)$ e no estado da Paraíba $(13,0 \%)^{4}$, e semelhante à encontrada em pesquisas de âmbito local desenvolvidas com famílias das três regiões de saúde reconhecidamente de baixa condição socioeconômica de Campinas-SP $(35,0 \%)^{27}$, de crianças assitidas em creches públicas municipais de Campina Grande-PB $(31,6 \%)^{6}$ e de classe socioeconômica baixa atendidas pela ESF da Regional Sudeste de Teresina-PI $(29,4 \%)^{28}$. Esses achados devem ser motivo de preocupação, uma vez que crianças que experimentam restrição alimentar quantitativa importante, ou episódios que configurem situação de fome, apresentam piores condições de saúde na infância ${ }^{13}$.

$\mathrm{Na}$ Árvore de Decisão, foi possível observar que maiores prevalências de insegurança alimentar moderada/grave entre as famílias beneficiárias do PBF, o que reforça achados anteriores com base em dados nacionais ${ }^{13,29}$ e estudos de abrangência local ${ }^{28,30}$. Ainda, converge com os resultados de uma revisão da literatura que apontou maiores chances de insegurança alimentar moderada entre usuários de serviços de saúde/ beneficiários do Programa Bolsa Família, quando comparado a populações de referência ${ }^{5}$. Esses achados sugerem o adequado destino dos recursos do PBF, entretanto sem conseguir melhorias na SAN ${ }^{5,6,9}$. Diante desse cenário, é fundamental salientar a importância de associar o benefício monetário a investimentos nos setores de saúde, educação, geração de trabalho e renda, com objetivo de reduzir a situação de vulnerabilidade familiar ${ }^{31}$. Resultados similares também foram 
Nível de Segurança Alimentar e Nutricional

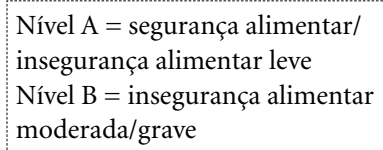

\begin{tabular}{|c|}
\hline Nó 0 \\
\hline Categoria $\mathrm{n} \%$ \\
\hline Nível A 27668,0 \\
\hline Nível B $130 \quad 32,0$ \\
\hline Total 406100,0 \\
\hline
\end{tabular}

Benefício do Programa Bolsa Família Valor $\mathrm{p}$ ajustado $=0,000$, Qui-quadrado $=26,890, \mathrm{df}=1$

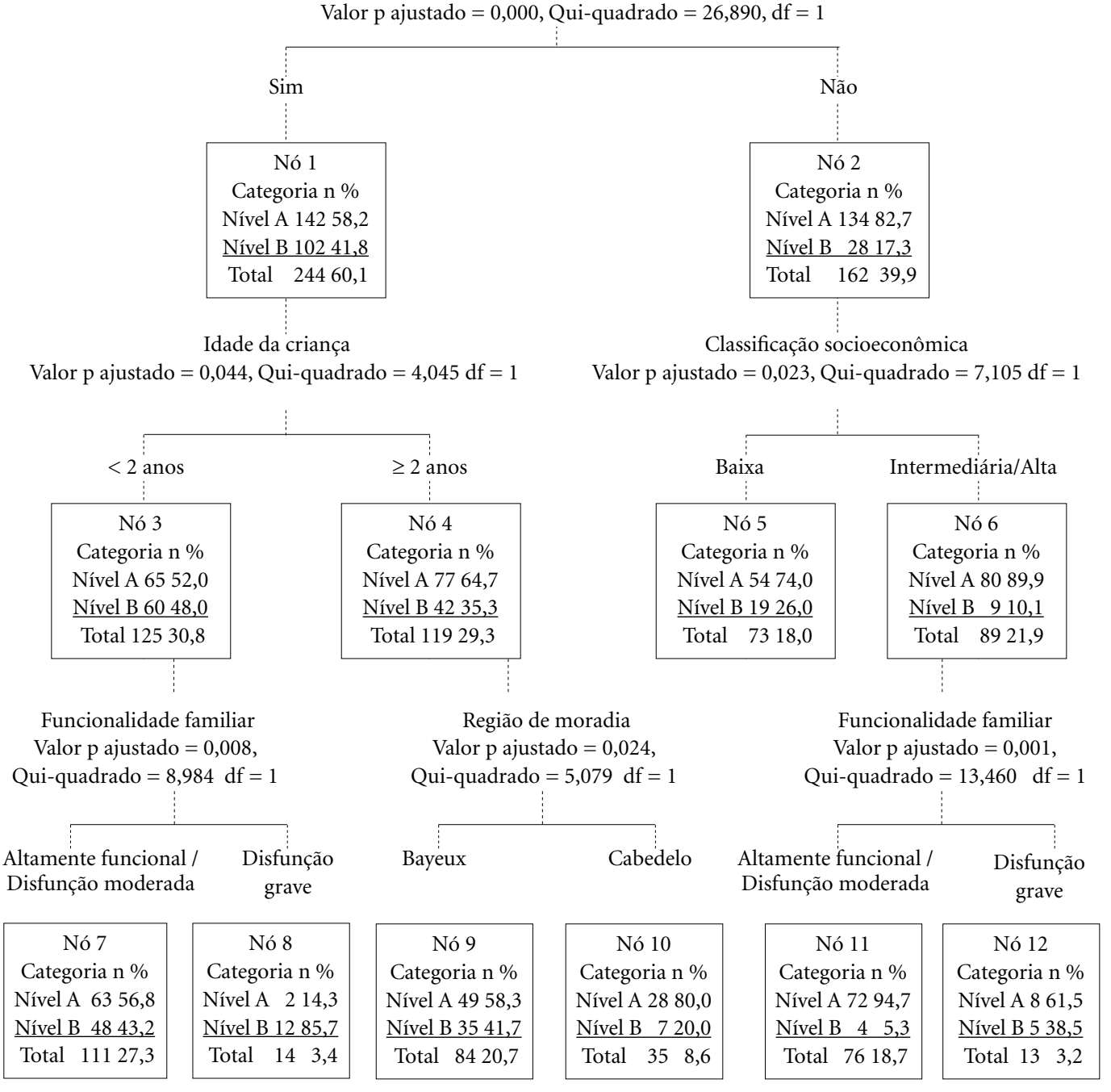

Df: degrees of freedom.

Figura 2. Análise multivariada por meio da Árvore de Decisão (CHAID) para a insegurança alimentar moderada/grave, ajustada pelos fatores investigados, em famílias com crianças menores de cinco anos residentes em dois municípios do estado da Paraíba, 2017.

registrados em relação ao PTCR do México, cujos autores reforçam possíveis consequências desses programas nas prevalências de sobrepeso/obesidade e, portanto, a inclusão de estratégias de pre- venção da obesidade como componente essencial dos mesmos ${ }^{32}$.

Em beneficiários do PBF, a maior probabilidade de insegurança alimentar moderada/grave 
ocorreu em núcleo familiar com criança menor de 2 anos. Estudo prévio com base em dados nacionais ressaltou entre famílias com crianças menores de 2 anos que ser beneficiário de PTCR priorizava famílias com insegurança alimentar moderada e grave ${ }^{33}$. Nesse sentido, recomendase a prática do aleitamento materno como forma de proteção à condição adversa de restrição e/ou privação alimentar e de promover a SAN dessas crianças $^{34}$. Adicionalmente, ressalta-se a importância da educação alimentar e nutricional que apoie a escolha de alimentos saudáveis, principalmente para as mulheres que são na maioria as gerenciadoras da alimentação e dos cuidados infantis, bem como as titulares do $\mathrm{PBF}^{13}$.

Neste estudo, a caracterização da funcionalidade das famílias como disfunção grave ou moderada/grave apresentou-se relevante na condição de insegurança alimentar moderada/grave em domicílios com crianças menores de 2 anos, apoiados pelo PBF, e de nível socioeconômico intermediário/alto, sem o benefício do PBF, respectivamente. Apesar de uma literatura escassa e controversa sobre a temática ${ }^{11}$, esses resultados encontram explicações na importância do apoio social na quantidade e/ou qualidade da alimentação, seja por meio da provisão de recursos ou por proporcionar sentimentos positivos, como se sentir cuidada, amada ou ter alguém com quem contar, que podem melhorar a capacidade de lidar com eventos estressantes ${ }^{11,12}$. Além disso, situações de IAN igualmente podem ser enfrentadas ao se ter pessoas que possam fornecer informações e orientação ${ }^{12}$ e relações sociais e comunitárias, particularmente em termos de recursos, que ajudem os grupos vulneráveis ${ }^{11}$. Achados semelhantes aos deste estudo foram anteriormente encontrados em outras localidades do Brasil ${ }^{11,12}$.

Os resultados desta pesquisa, especificamente no grupo de não beneficiários do PBF, alinhamse aos de outros estudos que mostraram a determinação socioeconômica da IAN nas famílias brasileiras, como esperado ${ }^{6,27,28,31}$. De maneira coerente, resultados provenientes de análises da tendência e de fatores associados à IAN no Brasil em 2004, 2009 e 2013 evidenciaram a influência de variáveis relacionadas com a situação socioeconômica, bem como menores avanços entre as famílias dos estratos de maior vulnerabilidade social, econômica e demográfica ${ }^{2}$. Em uma revisão sistemática sobre a IAN em diferentes contextos epidemiológicos no Brasil, a variável que apresentou maior associação com esse desfecho foi a pior renda familiar e grupos em iniquidade social, reforçando a conjuntura social e eco- nômica relacionada à fome ${ }^{5}$. Assemelhando-se às circunstâncias antepostas para a população brasileira, em pesquisas conduzidas na França ${ }^{35}$ e no México ${ }^{32}$, situações socioeconômicas de maior vulnerabilidades foram constatadas entre famílias vivendo em IAN, considerando a posse de bens de consumo e um índice da situação socioeconômica, respectivamente. A importância do nível socioeconômico no poder de compra, inclusive de alimentos, explica a relação da IAN com diversos determinantes domiciliares de cunho social ${ }^{2}$ e sua utilidade como indicador de iniquidade $^{5}$.

O município de residência também influenciou nas prevalências de IAN, possivelmente explicado pelas condições de vida mais precárias da população estudada residente na cidade com pior desenvolvimento humano ${ }^{30}$. O município de Bayeux (índice de desenvolvimento humano municipal médio de 0,649 e renda per capita de $\mathrm{R} \$$ $376,74)$ caracteriza-se por ter indicadores socioeconômicos mais desfavoráveis quando comprado ao de Cabedelo (índice de desenvolvimento humano municipal alto de 0,748 e renda per capita de R\$1.036,21) (http://www.atlasbrasil.org. br/2013/, acessado em 01/Mai/2019).

Como limitações do estudo pode ser destacado o receio que algumas famílias têm em responder às perguntas da EBIA. Contudo, à medida que conheciam os objetivos e a importância da pesquisa, os participantes sentiam maior segurança para responder aos entrevistadores. Além disso, a EBIA é um instrumento de fácil compreensão amplamente utilizado em pesquisas, inclusive em inquéritos nacionais, o que permite comparações com os dados da literatura.

\section{Conclusão}

Os resultados do presente estudo mostram que sete em cada dez famílias com crianças menores de 5 anos usuárias da ESF viviam em IAN, das quais, a cada dez, três encontravam-se em insegurança alimentar moderada/grave. Entre essas famílias, a insegurança alimentar moderada/ grave relaciona-se de forma mais relevante com o benefício do PBF, sugerindo que o Programa beneficia as famílias de maneira correta, mas sem conseguir melhorar o acesso à alimentação. Além disso, destacaram-se outros determinantes domiciliares da SAN como a presença no núcleo familiar de crianças menores de 2 anos, a disfuncionalidade familiar e o menor nível socioeconômico. 


\section{Colaboradores}

EES Santos, MM Oliveira e IM Bernardino participaram da análise e interpretação dos dados, redação e revisão final do artigo. D Figueroa Pedraza participou da elaboração do protocolo de estudo, concepção do artigo, análise e interpretação dos dados, redação e revisão final do artigo.

\section{Referências}

1. Organización de las Naciones Unidas para la Alimentación y la Agricultura (FAO), Fondo Internacional de Desarrollo Agrícola (FIDA), United Nations International Children's Emergency Fund (UNICEF), Programa Mundial de Alimentos (PMA), Organización Mundial de la Salud (OMS). El estado de la seguridad alimentaria y la nutrición en el mundo. Fomentando la resiliencia climática en aras de la seguridad alimentaria y la nutrición. Roma: FAO, FIDA, UNICEF, PMA, OMS; 2018.

2. Santos TG, Silveira JAC, Longo-Silva G, Ramires EKNM, Menezes RCE. Tendência e fatores associados à insegurança alimentar no Brasil: Pesquisa Nacional por Amostra de Domicílios 2004, 2009 e 2013. Cad Saude Publica 2018; 34(4):e00066917.

3. Brasil. Secretaria de Governo da Presidência da República, Ministério do Planejamento, Desenvolvimento e Gestão (MP). Relatório Nacional Voluntário sobre os Objetivos de Desenvolvimento Sustentável: Brasil 2017. Brasília: MP; 2017.

4. Instituto Brasileiro de Geografia e Estatística (IBGE). Pesquisa Nacional por Amostra de Domicílios: segurança alimentar 2013. Rio de Janeiro: IBGE; 2014.

5. Bezerra TA, Olinda RA, Figueroa Pedraza D. Insegurança alimentar no Brasil segundo diferentes cenários sociodemográficos. Cien Saude Colet 2017; 22(2):637651.

6. Pedraza FD, Gama JSFA. Segurança alimentar e nutricional de famílias com crianças menores de cinco anos do município de Campina Grande, Paraíba. Rev Bras Epidemiol 2015; 18(4):906-917.

7. Organização das Nações Unidas (ONU). Comissão Econômica para a América Latina e Caribe (CEPAL). Desenvolvimento sustentável na América Latina e no Caribe: Seguimento da Agenda das Nações Unidas para o Desenvolvimento Pós-2015 e Rio+20. Santiago: ONU; 2013.

8. Groot R, Palermo T, Handa S, Ragno LP, Peterman A. Cash Transfers and Child Nutrition: Pathways and Impacts. Development Policy Review 2017; 35(5):621643.

9. Wolf MR, Barros Filho AA. Estado nutricional dos beneficiários do Programa Bolsa Família no Brasil - uma revisão sistemática. Cien Saude Colet 2014; 19(5):1331-1338.

10. Kepple AW, Segall-Corrêa AM. Conceituando e medindo segurança alimentar e nutricional. Cien Saude Colet 2011; 16(1):187-199.

11. Interlenghi GS, Salles-costa R. Inverse association between social support and household food insecurity in a metropolitan area of Rio de Janeiro, Brazil. Public Health Nutr 2015; 18(16):2925-2933.

12. Santos IN, Damião JJ, Fonseca MJMD, Cople Rodrigues CDS, Aguiar OB. Food insecurity and social support in families of children with sickle-cell disease. J Pediatr 2019; 95(3):306-313.

13. Poblacion AP, Marín-León L, Segall-Corrêa NA, Silveira JAC, Taddei JAAC. Insegurança alimentar em domicílios brasileiros com crianças menores de cinco anos. Cad Saude Publica 2014; 30(5):1067-1078. 
14. Brasil. Portaria no 2.706 , de 18 de outubro de 2017 . Lista os municípios que finalizaram a adesão ao Programa Saúde na Escola para o ciclo 2017/2018 e os habilita ao recebimento do teto de recursos financeiros pactuados em Termo de Compromisso e repassa recursos financeiros para Municípios prioritários para ações de prevenção da obesidade infantil com escolares. Diário Oficial da União 2017; 20 out.

15. Brasil. Ministério da Saúde (MS). Atenção ao pré-natal de baixo risco. Brasília: MS; 2013. [Cadernos de Atenção Básica, 32].

16. Brasil. Ministério da Saúde (MS). Orientações para a coleta e análise de dados antropométricos em serviços de saúde: Norma Técnica do Sistema de Vigilância Alimentar e Nutricional - SISVAN. Brasília: MS; 2011.

17. Smilkstein G. The family APGAR: a proposal for a family function test and its use by physicians. J Fam Pract 1978; 6(6):1231-1239.

18. Good MV, Smilkstein G, Good BJ, Shaffer T, Arons T. The Family APGAR Index: A Study of Construct Validity. J Fam Pract 1979; 8(3):577-582.

19. Associação Brasileira de Empresas de Pesquisa (ABEP). Critério de classificação econômica Brasil. São Paulo: ABEP; 2016.

20. Segall-Corrêa AM, Marin-León L, Melgar-Quiñonez $\mathrm{H}$, Pérez-Escamilla R. Refinement of the Brazilian Household Food Insecurity Measurement Scale: Recommendation for a 14-item EBIA. Rev Nutr 2014; 27(2):241-251.

21. Soárez PC, Soares MO, Novaes HMD. Modelos de decisão para avaliações econômicas de tecnologias em saúde. Cien Saude Colet 2014; 19(10):4209-4222.

22. Speybroeck N. Classification and regression trees. Int J Public Health 2012; 57(1):243-246.

23. Hair Junior JF, Black WC, Babin BJ, Anderson RE, Tatham R. Análise multivariada de dados. $6^{a}$ ed. Porto Alegre: Bookman; 2009.

24. Lopes AF, Frota MTBA, Leone C, Szarfarc SC. Perfil nutricional de crianças no estado do Maranhão. Rev Bras Epidemiol 2019; 22:e190008.

25. Cuevas-Nasu L, Rivera-Dommarco JA, Shamah-Levy T, Mundo-Rosas V, Méndez-Gómez Humarán I. Inseguridad alimentaria y estado de nutrición en menores de cinco años de edad en México. Salud Publ Mex 2014; 56(Supl. 1):S47-53.

26. Betebo B, Ejajo T, Alemseged F, Massa D. Household Food Insecurity and Its Association with Nutritional Status of Children 6-59 Months of Age in East Badawacho District, South Ethiopia. J Environ Public Health 2017; 2017:6373595.

27. Souza BFNJ, Marin-Leon L, Camargo DFM, Segall-Corrêa AM. Demographic and socioeconomic conditions associated with food insecurity in households in Campinas, SP, Brazil. Rev Nutr 2016; 29(6):845-857.

28. Sabóia RCB, Santos MM. Prevalence of food insecurity and associated factors in households covered by the Family Health Strategy in Teresina-PI, Brazil, 20122013. Epidemiol Serv Saude 2015; 24(3):749-758.
29. Facchini LA, Nunes BP, Motta JVS, Tomasi E, Silva SM, Thumé E, Silveira DS, Siqueira FV, Dilélio AS, Saes MO, Miranda VIA, Volz PM, Osório A, Fassa AG. Insegurança alimentar no Nordeste e Sul do Brasil: magnitude, fatores associados e padrões de renda per capita para redução das iniquidades. Cad Saude Publica 2014; 30(1):161-174.

30. Peixoto MRG, Ramos K, Martins KA, Schincaglia RM, Braudes-Silva LA. Insegurança alimentar na área de abrangência do Núcleo de Apoio à Saúde da Família em Itumbiara, Goiás. Epidemiol Serv Saude 2014; 23(2):327-336.

31. Monteiro F, Schmidt ST, Costa IB, Almeida CCB, Matuda NS. Bolsa Família: insegurança alimentar e nutricional de crianças menores de cinco anos. Cien Saude Colet 2014; 19(5):1347-1358.

32. Shamah-Levy T, Rosas VM, Morales-Juan C, Cuevas-Nasu L, Méndez-Gómez-Humarán, Pérez-Escamilla R. Food insecurity and maternal-child nutritional status in Mexico: cross-sectional analysis of the National Health and Nutrition Survey 2012. BMJ Open 2017; 7(7):e014371.

33. Vega JB, Taddei JAAC, Poblacion AP. Características sociodemográficas e nutricionais de crianças brasileiras menores de 2 anos beneficiárias de programas de transferência condicionada de renda em 2006. Cien Saude Colet 2014; 19(3):931-942.

34. Gomes GP, Gubert MB. Aleitamento materno em crianças menores de 2 anos e situação domiciliar quanto à segurança alimentar e nutricional. J Pediatr 2012; 88(3):279-282.

35. Bocquier A, Vieux F, Lioret S, Dubuisson C, Caillavet F, Darmon N. Socio-economic characteristics, living conditions and diet quality are associated with food insecurity in France. Public Health Nutr 2015; 18(6):2952-2961.

Artigo apresentado em 30/05/2019

Aprovado em 07/08/2019

Versão final apresentada em 15/11/2019 
